\title{
MicroRNA-146a/NAPDH oxidase4 decreases reactive oxygen species generation and inflammation in a diabetic nephropathy model
}

\author{
RONG JUN WAN and YUE HONG LI \\ Department of Urology, Hospital of Tianjin Nankai, Nankai, Tianjin 300100, P.R. China
}

Received May 14, 2016; Accepted March 30, 2017

DOI: $10.3892 / \mathrm{mmr} .2018 .8407$

\begin{abstract}
The present study investigated the role of microRNA (miR)-146a in a diabetic nephropathy (DN) model, and its molecular mechanism. DN mice were given intraperitoneal injections of streptozotocin $(55 \mathrm{mg} / \mathrm{kg} /$ day) for 5 consecutive days as an in vivo DN model. The HK-2 human kidney cell line were exposed to $45 \%$ D-glucose as an in vitro DN model. Firstly, it was demonstrated that miR-146a expression was inhibited and NAPDH oxidase 4 (Nox4) was increased in DN mice. In HK-2 cells, overexpression of miR-146a inhibited Nox4 protein expression and decreased reactive oxygen species (ROS) generation, oxidative stress and inflammation, and suppressed vascular cell adhesion molecule-1 (VCAM-1) and intracellular adhesion molecule-1 (ICAM-1) protein expression. Nacetylcysteine, a Nox4 inhibitor, was demonstrated to inhibit ROS generation, suppress VCAM-1 and ICAM-1 protein expression, and decrease oxidative stress and inflammation in HK-2 cells following overexpression of miR-146a. In conclusion, these results indicated that miR-146a/Nox4 decreases ROS generation and inflammation and prevents DN. Therefore, miR-146a may represent a novel anti-inflammatory and -oxidative modulator of DN.
\end{abstract}

\section{Introduction}

Diabetic nephropathy (DN) is the most common complication of diabetes, as one of the main causes of renal failure. Its incidence has increased yearly, which has become a serious global public health problem (1). According to the estimation from the World Trade Organization, the global diabetes incidence will reach 370 million by 2025 , of which $30 \%$ will develop into DN (2). Pathological features primarily include glomerular hypertrophy, hyperplasia, a thickened basement

Correspondence to: Dr Rong Jun Wan, Department of Urology, Hospital of Tianjin Nankai, 122 Three Weft Road, Nankai, Tianjin 300100, P.R. China

E-mail: j13340@163.com

Key words: microRNA-146a, NAPDH oxidase 4, diabetic nephropathy, anti-inflammatory, anti-oxidative membrane, increase of extracellular matrix components, and gradual progression to glomerular sclerosis, interstitial fibrosis and loss of function, which eventually leads to chronic renal failure, reducing the quality of life of the patient (3). The morbidity of DN has many causes, and the pathogenesis is complex, which remains to be fully elucidated.

The function of a large number of microRNAs (miRNAs) have been studied in vivo. One-third of human genes are subject to the regulation of miRNAs (4). They are involved in various physiological and biochemical processes, including cell growth, differentiation, apoptosis, sugar metabolism, fat metabolism, insulin secretion, brain formation, cardiogenesis and stem cell differentiation, and are closely associated with the occurrence of many diseases (including cancer) (5). Different from other genes, miRNAs have an independent transcription method, of which the product is not translated into a protein; they identify specific targets and bond with them, thereby regulating target expression after transcription (6). A large number of studies have indicated that miRNAs are involved in the development of DN, and form a very complex network (7). The identification of miRNAs provides a novel way to study the pathogenesis of DN (8).

At present, the pathogenesis of DN remains to be fully elucidated. However, it is understood that the significant pathological features at early stages are glomerular hypertrophy caused by glomerular mesangial cell proliferation, and the elevated glomerular filtration rate induced by mesangial cell (MC) systolic dysfunction (9). Oxidative stress is enhanced in the state of high glucose, and the generation of reactive oxygen species (ROS) is increased (10). Nicotinamide adenine dinucleotide phosphate (NADPH) oxidase is the main source of ROS in MCs, and NAPDH oxidase 4 (Nox4) is the most important NADPH oxidase subunit in MCs (9). NADPH oxidase and Nox4-derived ROS is involved in the abnormal proliferation of MCs induced by high glucose (11).

Previous study have suggested that DN is a low-grade inflammatory disease, proposing an 'inflammatory theory' of diabetes. Inflammation is considered to promote the pathogenesis of diabetes, with significance in the development of diabetic complications including DN (12). Previous have shown that during the development of DN, inflammation is obvious, renal tissue is accompanied with the infiltration of various inflammatory cells, including mononuclear macrophages, and significant increase in the concentration of various 
pro-inflammatory cytokines can often be detected in the tissues and circulating blood $(12,13)$. The control of diabetes kidney inflammation is helpful for delaying the development of DN. Therefore, the oxidative stress of DN and inflammation are considered as a whole in current research, in which a large amount of active oxygen generated by enhanced oxidative stress caused by a high glucose environment is regarded as a major cause of DN inflammation, and inflammation increases oxidative stress in turn, indicating a significant interaction between them $(14,15)$. Therefore, the present study aimed to investigate whether miRNA-146a/Nox4 decreases ROS generation and inflammation in a DN model.

\section{Materials and methods}

Animal studies. The animal experimental protocol was approved by the Institutional Ethics Committee on Animal Care and Experimentation at the Hospital of Tianjin Nankai (Tianjin, China). Male C57BL/6 mice (age, 8 weeks; weight, 20-25 g) were obtained from the Animal Laboratory of Tianjin Nankai and maintained in a specific pathogen free environment with a $12-\mathrm{h}$ light/dark cycle at $22-24^{\circ} \mathrm{C}$ and $55-60 \%$ humidity and free access to food and water. All mice were randomly distributed into two groups: Control $(n=6)$ and DN model $(n=6)$. DN mice were given intraperitoneal injections of streptozotocin (STZ; 55 mg/kg/day; Sigma-Aldrich; Merck MGaA, Darmstadt, Germany) for 5 consecutive days as the DN model. Control mice were given intraperitoneal injections of saline for 5 consecutive days. All mice were sacrificed using decollation under anesthesia ( $35 \mathrm{mg} / \mathrm{kg} /$ day of pentobarbital sodium) and renal tissue samples were collected, washed PBS and stores at $-80^{\circ} \mathrm{C}$.

Cell culture and transfection. HK-2 human kidney cells were purchased from Type Culture Collection of the Chinese Academy of Sciences (Shanghai, China) and cultured in keratinocyte serum-free media (Invitrogen; Thermo Fisher Scientific, Inc., Waltham, MA, USA) supplemented with $100 \mathrm{U} / \mathrm{ml}$ penicillin $\mathrm{G}, 100 \mu \mathrm{g} / \mathrm{ml}$ streptomycin and $0.25 \mu \mathrm{g} / \mathrm{ml}$ amphotericin $\mathrm{B}$, and incubated at $37^{\circ} \mathrm{C}$ in a $100 \%$ humidified incubator containing $5 \% \mathrm{CO}_{2}$. The groups were as follows: DN model and D-glucose control, exposed to $45 \mathrm{D}$-glucose; control, exposed to D-glucose; negative, exposed to $5 \% \mathrm{D}$-glucose and transfected with a negative control plasmid; miR-146, exposed to $45 \% \mathrm{D}$-glucose and transfected with an miR-146 mimic and treated with nacetylecysteine (NAC, $3 \mathrm{mM}$, Beyotime Institute of Biotechnology, Haimen, China). The miR-146 mimic $(30 \mathrm{nM})$ and negative control duplex mimic $(30 \mathrm{nM})$ plasmids were transfected into HK-2 cells using Lipofectamine 2000 (Invitrogen; Thermo Fisher Scientific, Inc.).

Reverse transcription-quantitative polymerase chain reaction (RT-qPCR). Total RNA was extracted from HK-2 cells transfected with mimics using TRIzol reagent (Invitrogen; Thermo Fisher Scientific, Inc.) and then cDNA was synthesized using a Takara RNA PCR kit (Baoshengwu, Dalian, China). The gene expression of microRNA-146a and Nox4 were measured by qPCR using a SYBR Select Master mix (Bio-Rad Laboratories, Inc., Hercules, CA, USA). The primers used were as follows: Forward, 5-CTCTGCTCCTCCTGT TCGAC-3 and reverse, 5-ACGACCAAATCCGTTGACTC-3 for GAPDH; forward, 5-GCTGACGTTGCATGTTTCAG-3 and reverse, 5-CGGGAGGGTGGGTATCTAA-3 for Nox4. A CFX96 real-time PCR system (Bio-Rad Laboratories, Inc.) was run at $50^{\circ} \mathrm{C}$ for $1 \mathrm{~min}$ and at $95^{\circ} \mathrm{C}$ for $5 \mathrm{~min}$, followed by 40 cycles at $95^{\circ} \mathrm{C}$ for $30 \mathrm{sec}$ and $60^{\circ} \mathrm{C}$ for $1 \mathrm{~min}$. miRNA expression was measured using the quantitation cycle $(\mathrm{Ct})$ according to the $\Delta \Delta \mathrm{Cq}$ method. $\Delta \Delta \mathrm{Cq}=\Delta \mathrm{Cq}$ (gene of interest) $-\Delta \mathrm{Cq}$ (endogenous control) $(16,17)$.

Assessment of ROS concentration. ROS levels were analyzed by an Oxiselect Intracellular ROS Assay kit (Cell Biolabs, Inc., San Diego, CA, USA). HK-2 cells were incubated with the fluorescent dye 2,7-dichlorodihydrofluorescein diacetate (DCFH-DA; Cell Biolabs, Inc.) for $20 \mathrm{~min}$ at $37^{\circ} \mathrm{C}$ and $5 \% \mathrm{CO}_{2}$ in the dark. ROS levels were analyzed at wavelengths of 485 and $525 \mathrm{~nm}$ using a fluorescence microscope (Eclipse Ti-S, Nikon Corporation, Tokyo, Japan) and analyzed using NIS-Elements Basic Research software version 4.10 (Nikon Corporation). Cellular levels of ROS were measured by means of a previously described (18) semi-quantitative DCFH-DA fluorescence technique, which can be used to track alterations in ROS concentration over time. Intracellular ROS expression levels were analyzed by an Oxiselect Intracellular ROS Assay kit (Cell Biolabs, Inc.), which measures ROS by employing the cell-permeable fluorogenic probe DCFH-DA.

Assessment of oxidative stress and inflammation. Mice were anaesthetized using $35 \mathrm{mg} / \mathrm{kg}$ pentobarbital sodium (Sigma-Aldrich; Merck KGaA) and sacrificed using decapitation. Serum was collected. The supernatants were separated by centrifugation at $500 \mathrm{x}$ g for $5 \mathrm{~min}$ at $4^{\circ} \mathrm{C}$ and stored at $-80^{\circ} \mathrm{C}$. Superoxide dismutase (SOD; cat. no. A001-3), malondialdehyde (MDA; cat. no. A003-1), nuclear factor (NF)- $\kappa$ B (cat. no. H202), tumor necrosis factor (TNF)- $\alpha$ (cat. no. H052), interleukin (IL)-6 (cat. no. H007) and IL-1 $\beta$ (cat. no. H002) levels were measured using rat ELISA kits (Nanjing Institute of Biological Engineering) at a wavelength of $450 \mathrm{~nm}$ using a microplate reader (BMG Labtech $\mathrm{GmbH}$, Ortenberg, Germany), according to the manufacturer's protocol.

Western blot analysis. Total proteins were lysed from renal tissue in radioimmunoprecipitation buffer (Beyotime Institute of Biotechnology) and protein concentrations (collected by centrifugation at $12,000 \mathrm{x}$ g for $10 \mathrm{~min}$ at $4^{\circ} \mathrm{C}$ ) and determined using an Enhanced Bicinchoninic Acid Protein Assay kit (Beyotime Institute of Biotechnology). Proteins $(50 \mu \mathrm{g})$ were separated by $8-12 \%$ SDS-PAGE and transferred onto a polyvinylidene difluoride membrane (EMD Millipore, Billerica, MA, USA). Membranes were blocked in TBS with Tween-20 (TBST) buffer containing 5\% non-fat dry milk. Following this, they were probed with Nox4 (cat. no. sc-55142), vascular cell adhesion molecule (VCAM)-1 (cat. no. sc-1504), intracellular adhesion molecule (ICAM)-1 (cat. no. sc-1511), all at a 1:500 dilution and purchased from Sigma-Aldrich; Merck KGaA, and GAPDH (cat. no. AG019, 1:5,000, Beyotime Institute of Biotechnology) primary antibodies at $4^{\circ} \mathrm{C}$ overnight. Blots were washed with TBST buffer and then incubated with anti-rabbit horseradish peroxidase-conjugated secondary antibodies (1:5,000; cat. no. sc-2030; Santa Cruz Biotechnology, Inc., Dallas, TX, USA) for $1 \mathrm{~h}$ at room temperature. Protein 
bands were detected using an Enhanced Chemiluminescence Hyperfilm (GE Healthcare Life Sciences, Little Chalfont, UK) and quantified using Bio-Rad gel imaging system 4.6 software (Bio-Rad Laboratories, Inc.).

Statistical analysis. Data are expressed as the mean \pm standard deviation using SPSS software version 17.0 (SPSS, Inc., Chicago, IL, USA). One-way analysis of variance and Student's Newman-Keuls test for comparisons were used to determine differences between control and experimental groups. $\mathrm{P}<0.05$ was considered to indicate a statistically significant difference.

\section{Results}

Expression of microRNA-146a and Nox4 in the DN model. As presented in Fig. 1A, the expression of microRNA-146a in DN model mice was reduced compared with the control group. However, the expression of Nox 4 mRNA in DN model mice was increased compared with the control group (Fig. 1B).

Overexpression of miR-146a on Nox4 expression in the DN model. The overexpression of miR-146a on the effect on Nox4 expression in DN mice was evaluated. As presented in Fig. 2A, there was a significant inhibition of miR-146a expression in the negative group, compared with the control group. An miR-146a mimic effectively increased miR-146a expression in HK-2 cells exposed to D-glucose. Then, there was a significant increase in Nox 4 protein expression levels in HK-2 cells exposed to D-glucose, compared with the control group (Fig. 2B and C). Overexpression of miR-146a significantly inhibited Nox4 protein expression in $\mathrm{HK}-2$ cells exposed to D-glucose, compared with the D-glucose group (Fig. 2B and C).

Overexpression of miR-146a on ROS generation and oxidative stress in the DN model. Whether overexpression of miR-146a affected ROS generation and oxidative stress in the DN model was investigated. As presented in Fig. 3A and B, there was a significant increase of ROS generation and MDA activity, respectively, in HK-2 cells exposed to D-glucose, compared with the control group. However, there was a significant decrease in SOD activity in HK-2 cells exposed to D-glucose, compared with the control group (Fig. 3C). However, overexpression of miR-146a significantly reversed these effects (Fig. 3).

Overexpression of miR-146a on inflammation in the DN model. To confirm whether overexpression of miR-146a affected inflammation in the DN model, NF- $\kappa \mathrm{B}, \mathrm{TNF}-\alpha, \mathrm{IL}-6$ and IL-1 $\beta$ activity were measured using ELISA kits. There was a significant increase of NF- $\kappa$ B (Fig. 4A), TNF- $\alpha$ (Fig. 4B), IL-6 (Fig. 4C) and IL-1 $\beta$ (Fig. 4D) activity in HK-2 cells exposed to D-glucose, compared with the control group. Overexpression of miR-146a significantly reversed this effect (Fig. 4).

Overexpression of miR-146a on VCAM-1 protein expression in the DN model. Overexpression of miR-146a on VCAM-1 protein expression in the DN model was examined by western blot analysis. As presented in Fig. 5, VCAM-1 protein expression levels were significantly increased in the negative group compared with the control group. Overexpression of miR-146a significantly reversed this effect.

Overexpression of miR-146a on ICAM-1 protein expression in the DN model. Overexpression of miR-146a on ICAM-1 protein expression in the DN model was examined by western blot analysis. As presented in Fig. 6, ICAM-1 protein expression levels were significantly increased in the negative group compared with the control group. Overexpression of miR-146a significantly reversed this effect.

Inhibition of Nox4 on ROS generation and oxidative stress in $D N$ following overexpression of miR-146a. Whether inhibition of Nox4 affects ROS generation and oxidative stress in DN following overexpression of miR-146a was examined. ROS generation was significantly increased in the negative group compared with the control group; however, overexpression of miR-146a ameliorated this effect. NAC significantly decreased ROS generation (Fig. 7A), increased SOD activity (Fig. 7B) and decreased MDA activity (Fig. 7C) in HK-2 cells exposed to D-glucose following overexpression of miR-146a, compared with the miR-146a group.

Inhibition of Nox4 on inflammation in the DN model following overexpression of miR-146a. Whether inhibition of Nox4 would affect inflammation in the DN model following overexpression of miR-146a was examined. Inhibition of Nox4 by NAC significantly inhibited NF- $\kappa$ B (Fig. 8A), TNF- $\alpha$ (Fig. 8B), IL-6 (Fig. 8C) and IL-1 $\beta$ (Fig. 8D) activity in HK-2 cells exposed to D-glucose following overexpression of miR-146a, compared with the compared with the miR-146a group.

Inhibition of Nox4 on VCAM-1 protein expression in the DN model following overexpression of miR-146a. Whether inhibition of Nox4 would affect VCAM-1 protein expression in the DN model following overexpression of miR-146a was examined. As presented in Fig. 9, inhibition of Nox4 significantly inhibited VCAM-1 protein expression in HK-2 cells exposed to D-glucose following overexpression of miR-146a, compared with the miR-146a group.

Inhibition of Nox4 on ICAM-1 protein expression in the $D N$ model following overexpression of miR-146a. Whether inhibition of Nox4 would affect ICAM-1 protein expression in the DN model following overexpression of miR-146a was examined. As presented in Fig. 10, inhibition of Nox4 significantly suppressed the protein expression of ICAM-1 in HK-2 cells exposed to D-glucose following overexpression of miR-146a, compared with compared with the miR-146a group.

\section{Discussion}

$\mathrm{DN}$ is one of the most common chronic microvascular complications of diabetes, of which the incidence rate increases with diabetes duration (2). Patients with partial DN may develop into end-stage renal disease, causing kidney failure and requiring a kidney transplant, and may lead to mortality (19). The results demonstrated that overexpression of miR-146a significantly decreased NF- $\kappa$ B, TNF- $\alpha$, IL- 6 and IL-1 $\beta$ activities in HK2 cells exposed to D-glucose. 
A

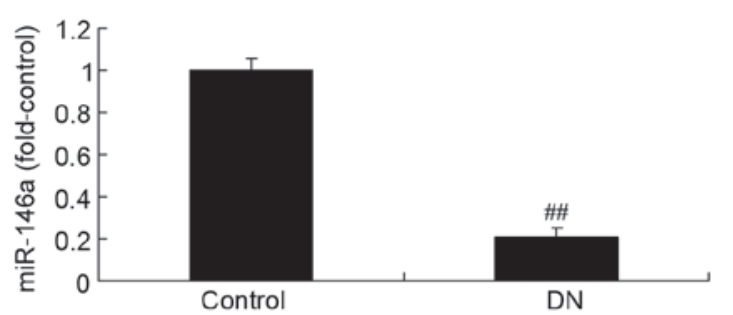

B

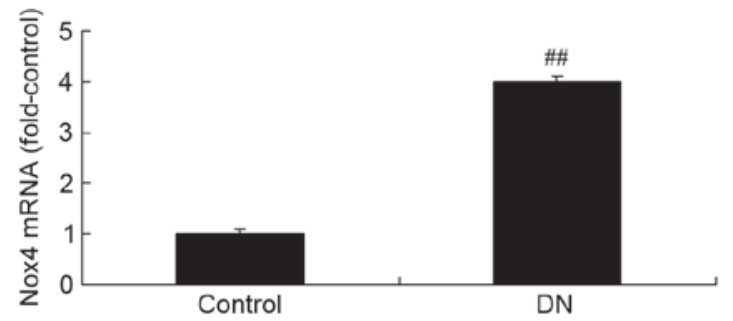

Figure 1. Expression of miR-146a and Nox4 in a DN model. mRNA expression levels of (A) miR-146a and (B) Nox4 in a DN model. Data are presented as the mean \pm standard deviation. ${ }^{\# /} \mathrm{P}<0.01$ vs. control group. DN, diabetic nephropathy; miR, microRNA; Nox4, NAPDH oxidase 4.
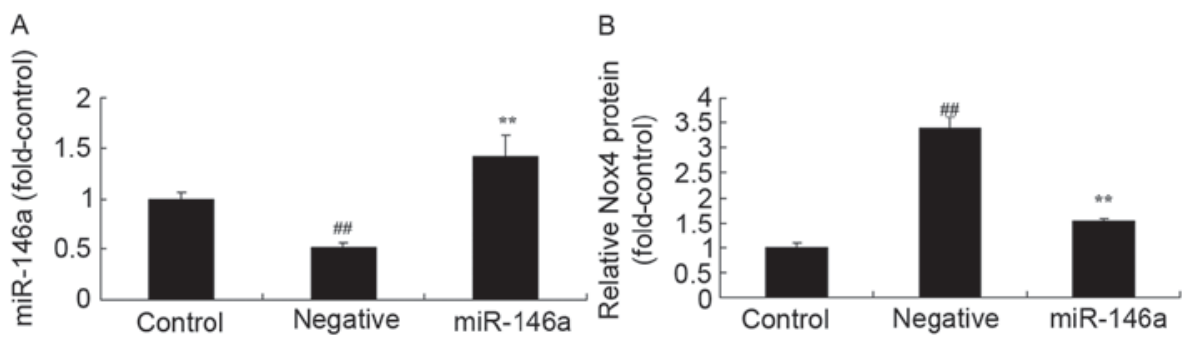

C

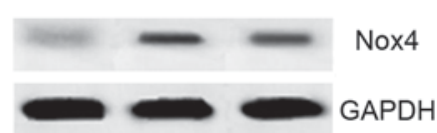

Control Negative miR-146a

Figure 2. Overexpression of miR-146a on Nox4 expression in a DN model. (A) miR-146a expression levels. (B) Quantification and (C) representative western blot images of Nox 4 protein expression levels. Data are presented as the mean \pm standard deviation. ${ }^{\# \#} \mathrm{P}<0.01$ vs. control group; ${ }^{* *} \mathrm{P}<0.01$ vs. negative group. DN, diabetic nephropathy; miR, microRNA; Nox4, NAPDH oxidase 4.
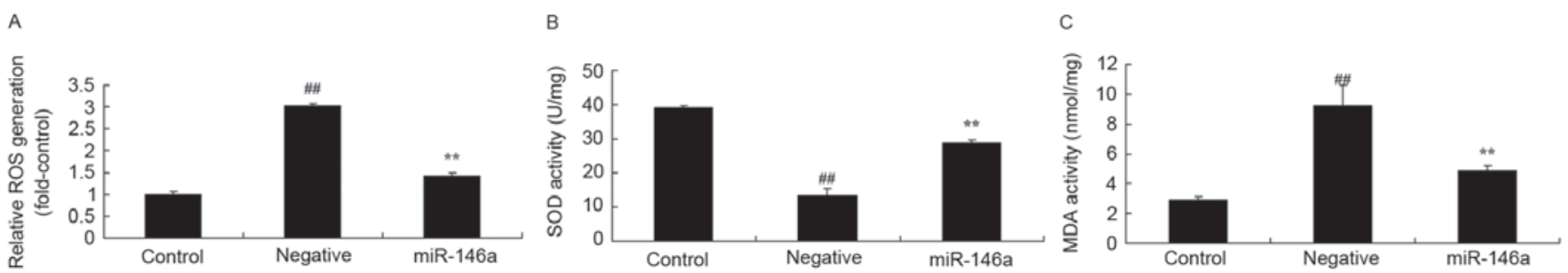

Figure 3. Overexpression of miR-146a on ROS generation and oxidative stress in a DN model. (A) ROS generation, and (B) SOD and (C) MDA activity in a DN model. Data are presented as the mean \pm standard deviation. ${ }^{\# \#} \mathrm{P}<0.01$ vs. control group; ${ }^{* * *} \mathrm{P}<0.01$ vs. negative group. DN, diabetic nephropathy; miR, microRNA; ROS, reactive oxygen species; SOD, superoxide dismutase; MDA, malondialdehyde.
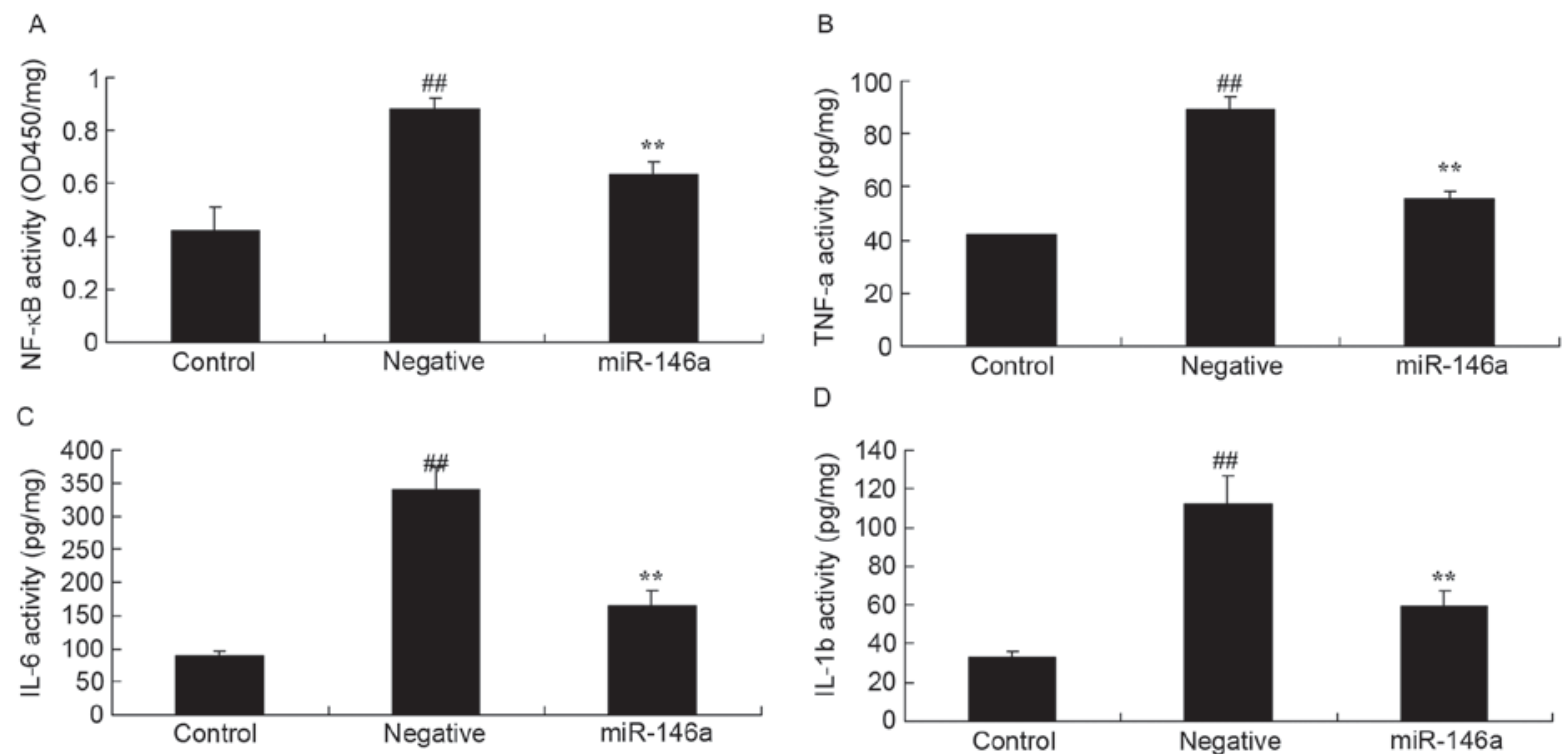

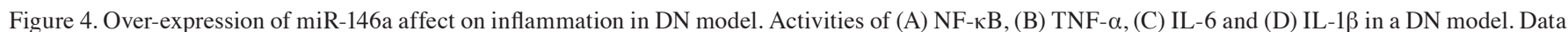
are presented as the mean \pm standard deviation. ${ }^{\# \#} \mathrm{P}<0.01$ vs. control group; ${ }^{* *} \mathrm{P}<0.01$ vs. negative group. $\mathrm{DN}$, diabetic nephropathy; miR, microRNA; NF- $\mathrm{kB}$, nuclear factor $\kappa \mathrm{B}$; TNF- $\alpha$, tumor necrosis factor- $\alpha$; IL, interleukin. 
A

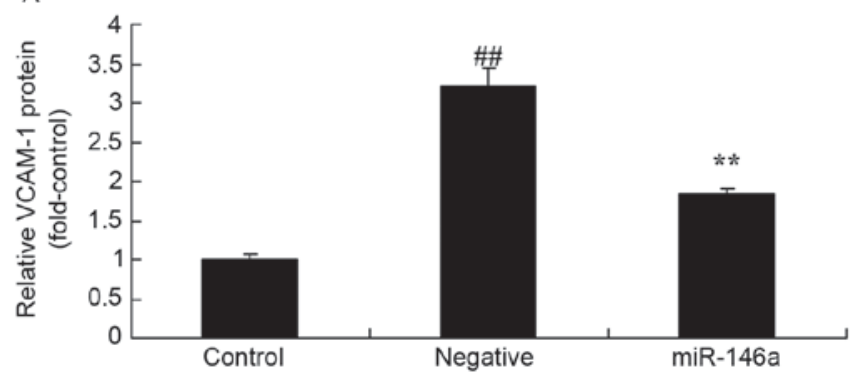

B

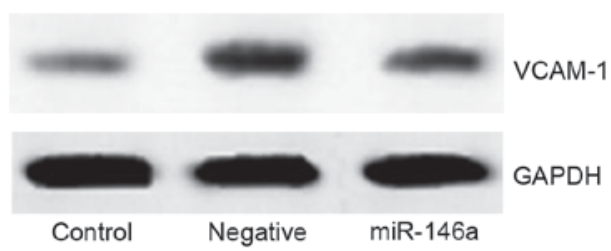

Figure 5. Overexpression of miR-146a on VCAM-1 protein expression in a DN model. (A) Quantification and (B) representative western blot images of VCAM-1 protein expression levels in a DN model. Data are presented as the mean \pm standard deviation. ${ }^{\# \#} \mathrm{P}<0.01$ vs. control group; ${ }^{* *} \mathrm{P}<0.01$ vs. negative group. DN, diabetic nephropathy; miR, microRNA; VCAM-1, vascular cell adhesion molecule-1. (VCAM)-1 (sc-1504), intracellular adhesion molecule.

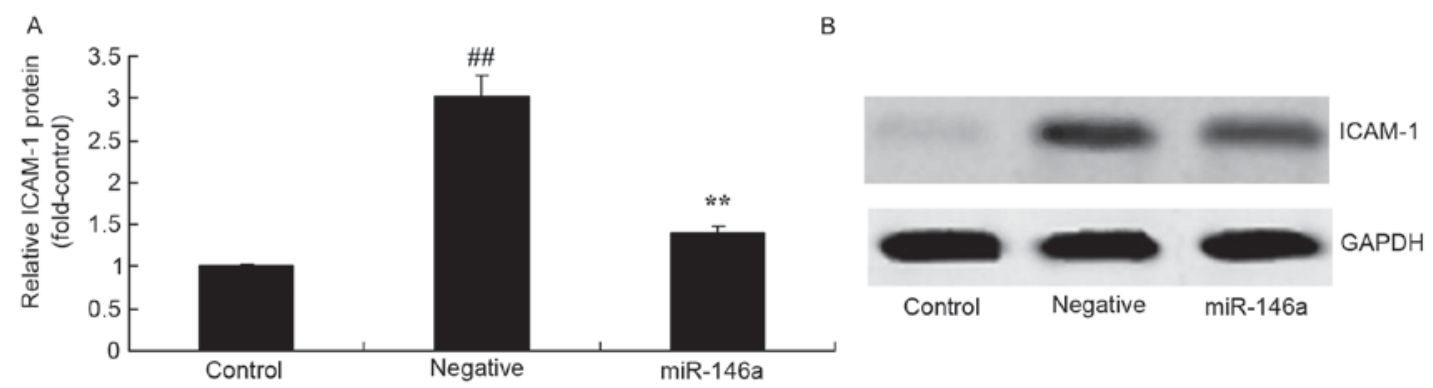

Figure 6. Overexpression of miR-146a on ICAM-1 protein expression in a DN model. (A) Quantification and (B) representative western blot images of ICAM-1 protein expression levels in a DN model. Data are presented as the mean \pm standard deviation. ${ }^{\# \#} \mathrm{P}<0.01$ vs. control group; ${ }^{* *} \mathrm{P}<0.01$ vs. negative group. DN, diabetic nephropathy; miR, microRNA; VCAM-1, vascular cell adhesion molecule-1.

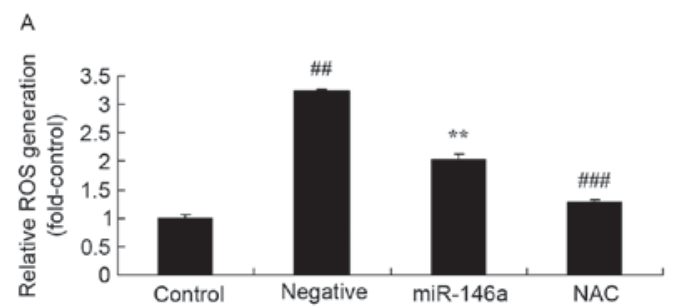

B

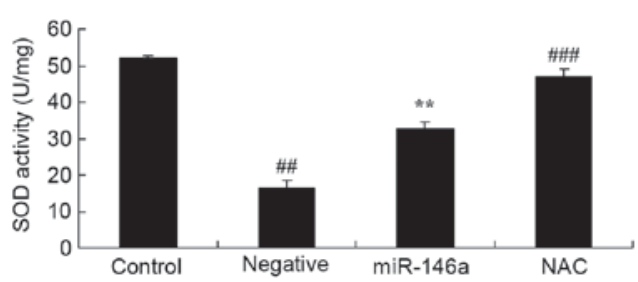

C

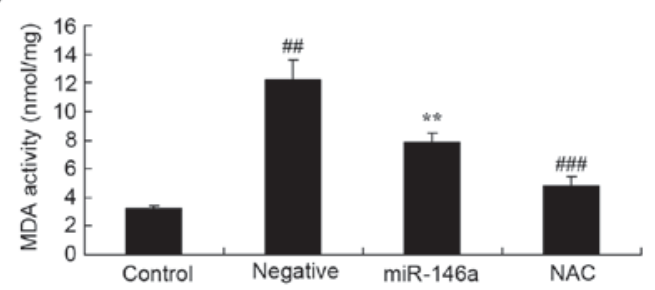

Figure 7. Inhibition of Nox4 on ROS generation and oxidative stress in DN following overexpression of miR-146a. Effect of treatment with the Nox4 inhibitor, NAC, on (A) ROS generation, and (B) SOD and (C) MDA activity in a DN model following overexpression of miR-146a. Data are presented as the mean \pm standard deviation. ${ }^{\# /} \mathrm{P}<0.01$ vs. control group; ${ }^{* *} \mathrm{P}<0.01$ vs. negative group; ${ }^{\# \#} \mathrm{P}<0.01$ vs. miR-146a group. $\mathrm{DN}$, diabetic nephropathy; miR, microRNA; NAC, nacetylcysteine; ROS, reactive oxygen species, SOD, superoxide dismutase; MDA, malondialdehyde; Nox4, NAPDH oxidase 4.
Increasing evidence suggests that there is an obvious enhancement in oxidative stress response under DM state (20). ROS produced in the oxidative stress reaction may be a major cause of vascular complications and DM (21). The excessive generation of ROS destroys the dynamic equilibrium of normal oxidation/reduction reaction, which results in oxidative damage of biological macromolecules, including proteins, lipids and nucleic acids, and interferes with regular biological activities (21). Certain studies have suggested that excessive generation of ROS is a direct result of hyperglycemia, and ROS generated from mitochondria by the induction of chronic hyperglycemia is the main initiator of multiple pathological pathways for DN $(22,23)$. The results of the present study suggested that overexpression of miR-146a significantly reversed ROS generation, SOD and MDA activity in HK-2 cells exposed to D-glucose.

NADPH oxidase is originally found in neutrophils, and also exists in other cell types such as endothelial, vascular smooth muscle, mesangial and renal tubular epithelial cells, similar to phagocytic cells, which also produce ROS under hyperglycemic conditions. In physiological conditions, NADPH oxidase activity is reduced, resulting in small amounts of ROS, and kidneys can tolerate this without injury (24). However, under the stimulation of various factors, including diabetes DNA oxidative damage, its activity is increased significantly. When hemodynamic alterations occur, excessive ROS can cause kidney cell dysfunction and even more serious injuries (25). Nox is a homolog of the catalytic subunit gp91phox, mainly including Nox1, Nox3, Nox4 and Nox5, and exist in a variety of non-phagocytic cells, with cell and tissue specificity, and can 

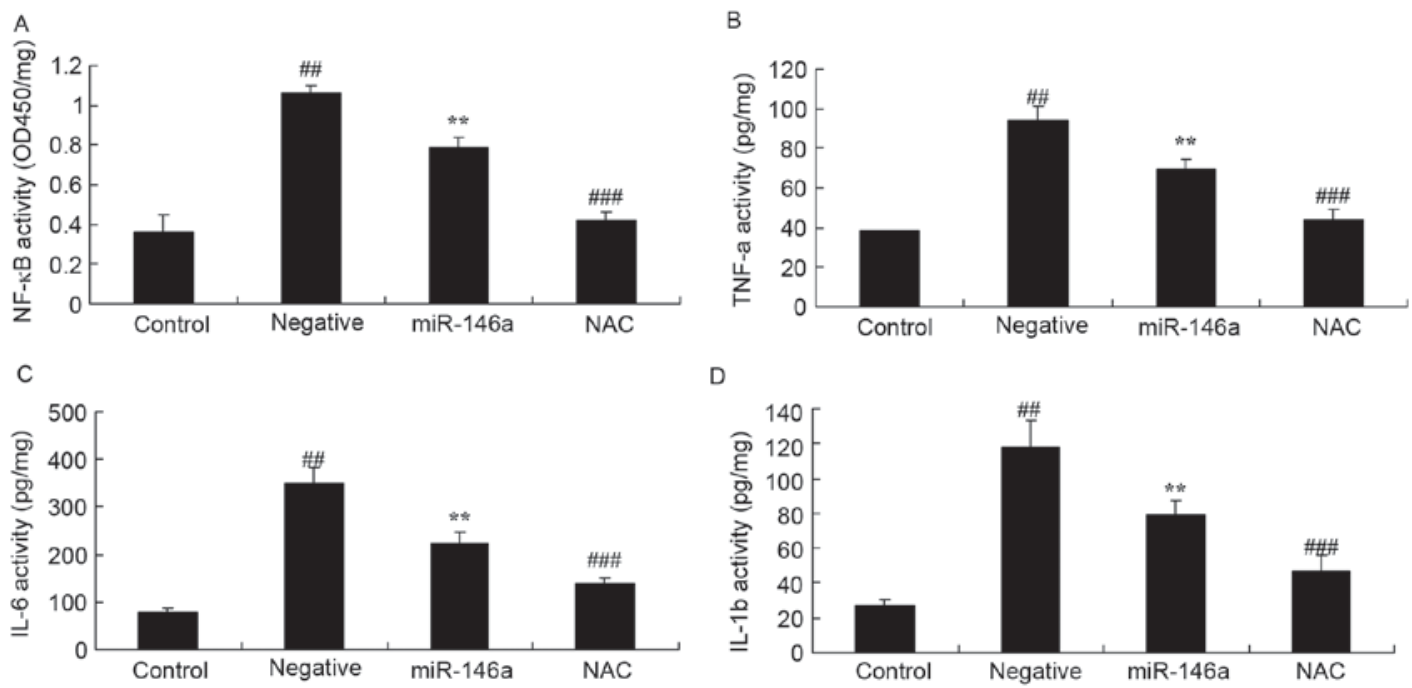

Figure 8. Inhibition of Nox 4 affect on inflammation in a DN model following overexpression of miR-146a. Effect of treatment with the Nox4 inhibitor, NAC, on (A) NF-KB, (B) TNF- $\alpha$, (C) IL-6 and (D) IL-1 $\beta$ activity in a DN model following overexpression of miR-146a. Data are presented as the mean \pm standard deviation. ${ }^{\# \#} \mathrm{P}<0.01$ vs. control group; ${ }^{* *} \mathrm{P}<0.01$ vs. negative group; ${ }^{\# \# "} \mathrm{P}<0.01$ vs. miR-146a group. DN, diabetic nephropathy; miR, microRNA; NAC, nacetylcysteine; NF-кB, nuclear factor $\kappa \mathrm{B}$; TNF- $\alpha$, tumor necrosis factor- $\alpha$; IL, interleukin; OD, optical density.

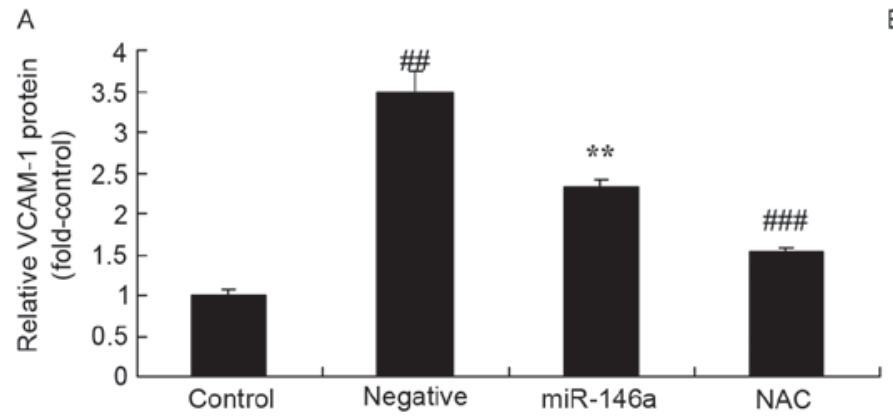

Figure 9. Inhibition of Nox4 on VCAM-1 protein expression in DN model following overexpression of miR-146a. (A) Quantification and (B) representative western blot images of ICAM-1 protein expression levels in a DN model, following treatment with the Nox4 inhibitor, NAC. Data are presented as the mean \pm standard deviation. ${ }^{\# \#} \mathrm{P}<0.01$ vs. control group; ${ }^{* *} \mathrm{P}<0.01$ vs. negative group; ${ }^{\# \#} \mathrm{P}<0.01$ vs. miR-146a group. DN, diabetic nephropathy; miR, microRNA; VCAM-1, vascular cell adhesion molecule-1; Nox4, NAPDH oxidase 4.
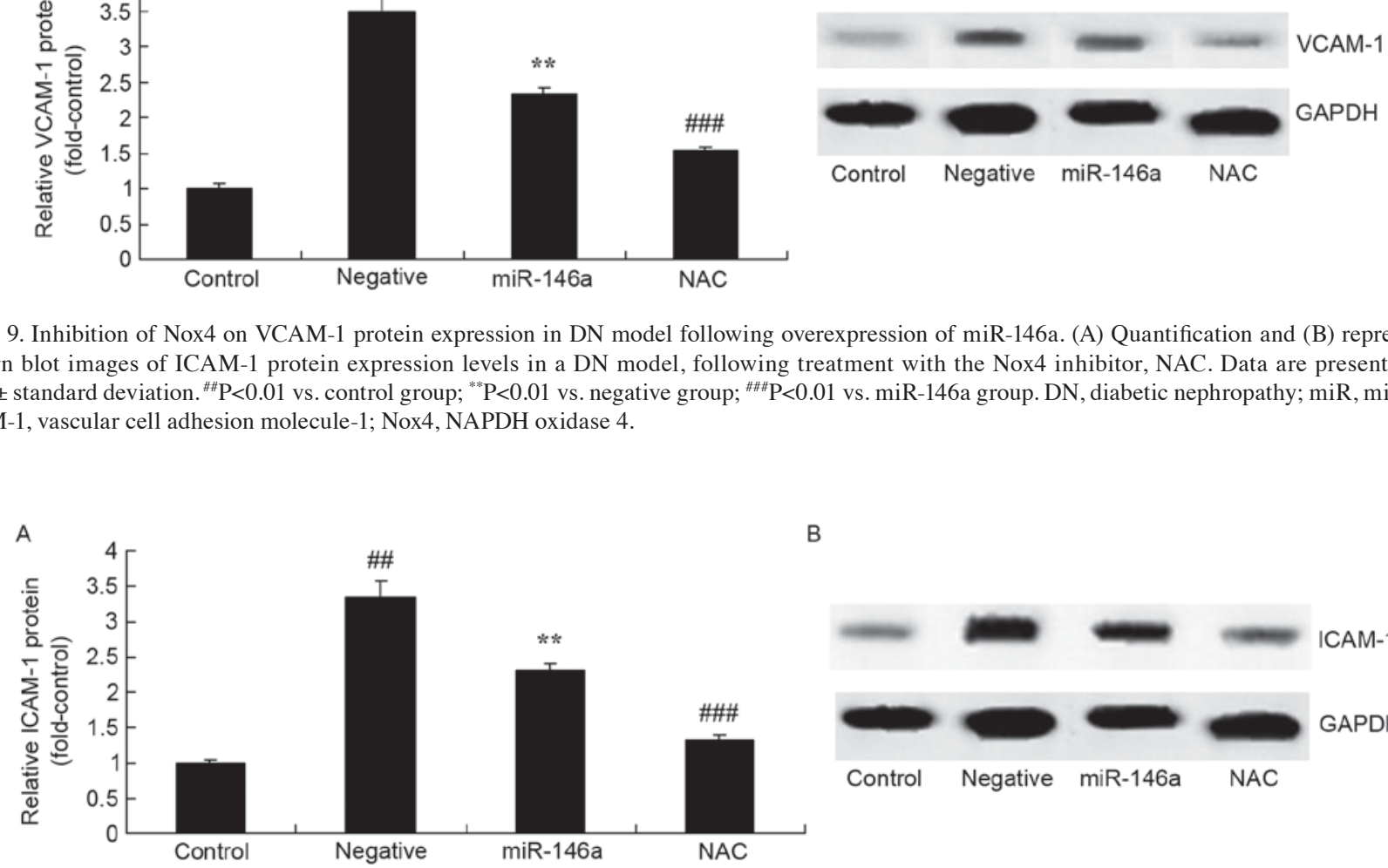

Figure 10. Inhibition of Nox4 on ICAM-1 protein expression in DN model following overexpression of miR-146a. (A) Quantification and (B) representative western blot images of ICAM-1 protein expression levels in a DN model, following treatment with the Nox4 inhibitor, NAC. Data are presented as the mean \pm standard deviation. ${ }^{\# \#} \mathrm{P}<0.01$ vs. control group; ${ }^{* *} \mathrm{P}<0.01$ vs. negative group; ${ }^{\# \# \#} \mathrm{P}<0.01$ vs. miR-146a group. DN, diabetic nephropathy; miR, microRNA; ICAM-1, intracellular adhesion molecule-1; Nox4, NAPDH oxidase 4.

generate ROS through catalysis (26). As a second messenger, ROS generated by Nox is involved in the regulation of cell proliferation, differentiation and apoptosis. Nox4 is the main type present in MCs in Nox subunits (27). The endogenous ROS of Nox4 is closely associated with glomerular injury associated with diabetes, and upregulation of Nox4 expression can induce glomerular hypertrophy in diabetic rat models (9). The results of the present study demonstrated that overexpression of miR-146a significantly inhibited Nox4 mRNA expression in HK-2 cells exposed to D-glucose. 
As the members of the immunoglobulin superfamily, VCAM-1 and ICAM-1 have increased expressions in DN, which are important in the DN immune response and inflammatory reaction, and their effect in DN has gained increasing attention (28). VCAM-1 and ICAM-1 are a class of glycoproteins distributed on the cell surface or extracellular matrix, with adhesive functions. They act by ligand-receptor interactions, which serves an important role in many physiological and pathological processes, including embryonic differentiation, maintenance of healthy structure, inflammation and immune response, blood coagulation and thrombosis, wound healing, and tumor spread and metastasis $(29,30)$. In the present study, overexpression of miR-146a significantly suppressed upregulated VCAM-1 and ICAM-1 protein expression levels in HK-2 cells exposed to D-glucose.

The early stage of DN primarily includes monocyte/macrophage infiltration, in which monocytes gathered in the kidney are activated and proliferate into macrophages, further inducing the production of the inflammatory cytokines, including TNF- $\alpha$, IL-6 and matrix metalloproteinase (15). Therefore, a series of circulating inflammatory markers such as TNF- $\alpha$ and IL-6 have increased expression levels in DN, which are closely associated with disease progression and proteinuria (31). In addition, hyperglycemia and angiotensin II stimulate vascular endothelial growth factor production, causing the release of endothelial nitric oxide, blood vessel dilation and increased glomerular filtration. Hyperglycemia also induces the synthesis of TGF- $\beta 1$, IV collagen and fibronectin, so as to increase the extracellular matrix (32). The results of the present study indicated that NAC, a ROS inhibitor, suppressed VCAM-1 and ICAM-1 protein expression, and decreased oxidative stress and inflammation of HK-2 cells following overexpression of miR-146a.

In conclusion, the present study demonstrated that overexpression of miR-146a significantly decreased NF- $\mathrm{kB}$, TNF- $\alpha$, IL- 6 and IL- $1 \beta$ activities, inhibited ROS generation and MDA activity, and increased SOD activity via Nox4/VCAM-1 and ICAM-1 expression in HK-2 cells exposed to D-glucose. Therefore, miR-146a/Nox4 may represent a potential therapeutic target for patients with DN.

\section{References}

1. Zilişteanu DS, Atasie T and Voiculescu M: Efficacy of long-term low-dose sulodexide in diabetic and non-diabetic nephropathies. Rom J Intern Med 53: 161-169, 2015.

2. Shima A, Miyamoto M, Kubota Y, Takagi G and Shimizu W: Beraprost sodium protects against diabetic nephropathy in patients with arteriosclerosis obliterans: A prospective, randomized, open-label study. J Nippon Med Sch 82: 84-91, 2015

3. Van Buren PN, Adams-Huet B, Nguyen M, Molina C and Toto RD: Potassium handling with dual renin-angiotensin system inhibition in diabetic nephropathy. Clin J Am Soc Nephrol 9: 295-301, 2014.

4. Peng R, Liu H, Peng H, Zhou J, Zha H, Chen X, Zhang L, Sun Y, Yin P, Wen L, et al: Promoter hypermethylation of let-7a-3 is relevant to its down-expression in diabetic nephropathy by targeting UHRF1. Gene 570: 57-63, 2015.

5. Kato M, Castro NE and Natarajan R: MicroRNAs: Potential mediators and biomarkers of diabetic complications. Free Radic Biol Med 64: 85-94, 2013.

6. Conserva F, Pontrelli P, Accetturo M and Gesualdo L: The pathogenesis of diabetic nephropathy: Focus on microRNAs and proteomics. J Nephrol 26: 811-820, 2013.
7. Alvarez ML and DiStefano JK: Towards microRNA-based therapeutics for diabetic nephropathy. Diabetologia 56: 444-456, 2013.

8. Silambarasan M, Tan JR, Karolina DS, Armugam A, Kaur C and Jeyaseelan K: MicroRNAs in hyperglycemia induced endothelial cell dysfunction. Int J Mol Sci 17: 518, 2016.

9. Ahmad A, Mondello S, Di Paola R, Mazzon E, Esposito E, Catania MA, Italiano D, Mondello P, Aloisi C and Cuzzocrea S: Protective effect of apocynin, a NADPH-oxidase inhibitor, against contrast-induced nephropathy in the diabetic rats: A comparison with n-acetylcysteine. Eur J Pharmacol 674: 397-406, 2012.

10. Jeong SI, Kim SJ, Kwon TH, Yu KY and Kim SY: Schizandrin prevents damage of murine mesangial cells via blocking NADPH oxidase-induced ROS signaling in high glucose. Food Chem Toxicol 50: 1045-1053, 2012

11. Winiarska K, Dzik JM, Labudda M, Focht D, Sierakowski B, Owczarek A, Komorowski L and Bielecki W: Melatonin nephroprotective action in Zucker diabetic fatty rats involves its inhibitory effect on NADPH oxidase. J Pineal Res 60: 109-117, 2016.

12. Jangale NM, Devarshi PP, Bansode SB, Kulkarni MJ and Harsulkar AM: Dietary flaxseed oil and fish oil ameliorates renal oxidative stress, protein glycation, and inflammation in streptozotocin-nicotinamide-induced diabetic rats. J Physiol Biochem 72: 327-336, 2016.

13. Borsting E, Patel SV, Decleves AE, Declèves AE, Lee SJ, Rahman QM, Akira S, Satriano J, Sharma K, Vallon V and Cunard R: Tribbles homolog 3 attenuates mammalian target of rapamycin complex-2 signaling and inflammation in the diabetic kidney. J Am Soc Nephrol 25: 2067-2078, 2014.

14. Deliyanti D, Zhang Y, Khong F, Berka DR, Stapleton DI, Kelly DJ and Wilkinson-Berka JL: FT011, a novel cardiorenal protective drug, reduces inflammation, gliosis and vascular injury in rats with diabetic retinopathy. PLoS One 10: e0134392, 2015.

15. Donate-Correa J, Martín-Núñez E, Muros-de-Fuentes M, Mora-Fernández C and Navarro-González JF: Inflammatory cytokines in diabetic nephropathy. J Diabetes Res 2015: 948417, 2015.

16. Wang TF, Lei Z, Li YX, Wang YS, Wang J, Wang SJ, Hao YJ, Zhou R, Jin SJ, Du J, et al: Oxysophoridine protects against focal cerebral ischemic injury by inhibiting oxidative stress and apoptosis in mice. Neurochem Res 38: 2408-2417, 2013.

17. Livak KJ and Schmittgen TD: Analysis of relative gene expression data using real-time quantitative PCR and the 2(-Delta Delta C(T)) method. Methods 25: 402-408, 2001

18. Muluye RA, Bian Y, Wang L, Alemu PN, Cui H, Peng X and Li S: Placenta peptide can protect mitochondrial dysfunction through inhibiting ROS and TNF- $\alpha$ generation, by maintaining mitochondrial dynamic network and by increasing il-6 level during chronic fatigue. Front Pharmacol 7: 328, 2016.

19. Schneider MP, Schneider A, Jumar A, Kistner I, Ott C and Schmieder RE: Effects of folic acid on renal endothelial function in patients with diabetic nephropathy: Results from a randomized trial. Clin Sci (Lond) 127: 499-505, 2014.

20. Moon JY, Tanimoto M, Gohda T, HagiwaraS, YamazakiT, OharaI, Murakoshi M, Aoki T, Ishikawa Y, Lee SH, et al: Attenuating effect of angiotensin-(1-7) on angiotensin II-mediated NAD(P)H oxidase activation in type 2 diabetic nephropathy of KK-A(y)/Ta mice. Am J Physiol Renal Physiol 300: F1271-F1282, 2011.

21. Wang JC, Zhao Y, Chen SJ, Long J, Jia QQ, Zhai JD, Zhang Q, Chen Y and Long HB: AOPPs induce MCP-1 expression by increasing ROS-mediated activation of the NF- $\kappa \mathrm{B}$ pathway in rat mesangial cells: Inhibition by sesquiterpene lactones. Cell Physiol Biochem 32: 1867-1877, 2013.

22. Pessôa BS, Peixoto EB, Papadimitriou A, Lopes de Faria JM and Lopes de Faria JB: Spironolactone improves nephropathy by enhancing glucose-6-phosphate dehydrogenase activity and reducing oxidative stress in diabetic hypertensive rat. J Renin Angiotensin Aldosterone Syst 13: 56-66, 2012.

23. Thallas-Bonke V, Thorpe SR, Coughlan MT, Fukami K, Yap FY, Sourris KC, Penfold SA, Bach LA, Cooper ME and Forbes JM: Inhibition of NADPH oxidase prevents advanced glycation end product-mediated damage in diabetic nephropathy through a protein kinase $\mathrm{C}$-alpha-dependent pathway. Diabetes 57 : 460-469, 2008

24. He T, Guan X, Wang S, Xiao T, Yang K, Xu X, Wang J and Zhao J: Resveratrol prevents high glucose-induced epithelial-mesenchymal transition in renal tubular epithelial cells by inhibiting NADPH oxidase/ROS/ERK pathway. Mol Cell Endocrinol 402: 13-20, 2015. 
25. Towler DA: Mitochondrial ROS deficiency and diabetic complications: AMP [K]-lifying the adaptation to hyperglycemia. J Clin Invest 123: 4573-4576, 2013.

26. Zhang L, Pang S, Deng B, Qian L, Chen J, Zou J, Zheng J, Yang L, Zhang $\mathrm{C}$, Chen $\mathrm{X}$, et al: High glucose induces renal mesangial cell proliferation and fibronectin expression through JNK/NF- $\mathrm{B} / \mathrm{NADPH}$ oxidase/ROS pathway, which is inhibited by resveratrol. Int J Biochem Cell Biol 44: 629-638, 2012.

27. Jha JC, Gray SP, Barit D, Okabe J, El-Osta A, Namikoshi T, Thallas-Bonke V, Wingler K, Szyndralewiez C, Heitz F, et al: Genetic targeting or pharmacologic inhibition of NADPH oxidase nox4 provides renoprotection in long-term diabetic nephropathy. J Am Soc Nephrol 25: 1237-1254, 2014.

28. Liu JJ, Yeoh LY, Sum CF, Tavintharan S, Ng XW, Liu S, Lee SB, Tang WE and Lim SC; SMART2D study: Vascular cell adhesion molecule-1, but not intercellular adhesion molecule-1, is associated with diabetic kidney disease in Asians with type 2 diabetes. J Diabetes Complications 29: 707-712, 2015.
29. Matsui T, Nishino Y, Maeda S, Takeuchi M and Yamagishi S: Irbesartan inhibits advanced glycation end product (AGE)-induced up-regulation of vascular cell adhesion molecule-1 (VCAM-1) mRNA levels in glomerular endothelial cells. Microvasc Res 81: 269-273, 2011.

30. Alkhalaf A, Kleefstra N, Groenier KH, Bilo HJ, Gans RO, Heeringa P, Scheijen JL, Schalkwijk CG, Navis GJ and Bakker SJ: Effect of benfotiamine on advanced glycation endproducts and markers of endothelial dysfunction and inflammation in diabetic nephropathy. PLoS One 7: e40427, 2012.

31. Jheng HF, Tsai PJ, Chuang YL, Shen YT, Tai TA, Chen WC, Chou CK, Ho LC, Tang MJ, Lai KT, et al: Albumin stimulates renal tubular inflammation through an HSP70-TLR4 axis in mice with early diabetic nephropathy. Dis Model Mech 8: 1311-1321, 2015.

32. Yaghobian D, Don AS, Yaghobian S, Chen X, Pollock CA and Saad S: Increased sphingosine 1-phosphate mediates inflammation and fibrosis in tubular injury in diabetic nephropathy. Clin Exp Pharmacol Physiol 43: 56-66, 2016. 